\title{
The Mooncup: A Tool to Measure Menstrual Bleeding Loss Volume
}

\author{
Barranco-Castillo Enriqueta ${ }^{1 *}$, Martos-García Raúl ${ }^{2,3}$ and Molina-Muñoz David ${ }^{4}$ \\ ${ }^{1}$ Department of Obstetric-Gynaecology, Granada University, Spain \\ ${ }^{2}$ Andalusian Regional Health Service, Priego de Cordoba Health Centre, Clinical Management Unit, Spain \\ ${ }^{3}$ Departament of Nursing, Spanish Red Cross University School of Sevilla University, Spain \\ ${ }^{4}$ Department of Statistics and Operational Research, Granada University, Spain
}

Submission: November 17, 2017; Published: January 22, 2018

*Corresponding author: Enriqueta Barranco-Castillo, Departamento de Obstetricia y Ginecología, Facultad de Medicina, Universidad de Granada, Avda. De la Innovación s/n, 18016 Granada, Spain, Tel: +34 653635761; Email: ebc@ugr.es

\begin{abstract}
Background: The mooncup is not a tool habitually used for quantifying the volume of menstrual blood loss.

objective: The aims of the study were to explore mooncup management by Spanish women and estimate the volume of menstrual blood loss.

Method: This is a comparative observational study involving 94 healthy women who participated voluntarily following a mass invitation on Face book. This is an accidental, non-probability sampling technique. The participants completed an online questionnaire drafted using the following program: https://docs.google.com/spreadsheets/u/0/, which included socio-demographic and mooncup use factors, with the aim of estimating menstrual volume. Depending on individual hygiene habits the cup was emptied when full, half-full or only stained.

Result: Overall, a mean figure of 3,25 cups/woman/day were emptied (SD 1.77, CI 95\%, 2.88-3.61). The average volume per woman and menstruation was 173 cc (SD 139.08, CI 95\%, 144.24-202.17). In 75\% of cases this figure was $\geq 88$ with a clot expulsion rate of $70 \%$. Bleeding
\end{abstract} lasted for an average of 4.86 days (SD 1.05, CI 95\% 4.62-5.10).

Conclusion and recommendation: The mooncup is a new method for determining menstrual blood volume. It has enabled us to establish that menstrual bleeding in $75 \%$ of the sample was over $80 \mathrm{cc}$ (average 173cc), which represents a risk of High Menstrual Bleeding, even in women with no known gynecological disease. More extensive population studies are needed to corroborate these findings and investigate their causes.

Keywords: Mooncup; Feminine hygiene; Menstruation; Blood volume

Abbreviations: PBAC: Pictorial Blood Assessment Chart; FIGO: International Federation of Gynecology and Obstetrics; MBL: Menstrual Blood Loss

\section{Introduction}

\section{Background}

The mooncup is not a tool used for quantifying menstrual volume, but, rather, a menstrual hygiene product which is increasingly used and Spain is no exception to this trend. A literature review enabled us to identify comparative studies regarding the advantages of mooncup use in comparison to sanitary pads and tampons (degree of comfort, user satisfaction etc), but authors have not focused on designing studies aiming to quantify menstrual volume in healthy mooncup users [13]. The advantages of the mooncup, in this sense, are to allow direct evaluation of volume, compared with the Pictorial Blood
Assessment Chart (PBAC), which is based on the absorption capacity of sanitary pads and tampons [4]. Some studies suggest that the use of mooncups amongst women in developing countries or with limited economic resources should be promoted as it is an economical, environment-friendly product, valid for management of menstruation [3]. Classical studies on this issue led the FIGO (2011) to establish a basic classification to determine whether menstrual bleeding was within normal parameters or not. According to these indications it should not exceed 80cc expelled in 4.5 - 8 days of bleeding [5-8], but in daily clinical practice it sometimes proves difficult to measure menstrual flow in these terms. 


\section{Global Journal of Reproductive Medicine}

\section{Objectives}

The objective of this study is to estimate the volume of menstrual bleeding in a sample of Spanish mooncup users.

\section{Methodology}

To fulfill our objective an observational case-comparison study was designed. An accidental, non-probability sampling technique was applied involving an appeal and mass recruitment of mooncup users through Facebook. The case collection period lasted from $1^{\text {st }}$ January until $31^{\text {st }}$ May 2015 . The users interested in participating contacted the researcher and were given information privately about inclusion criteria and conditions for participation. Once recruitment was confirmed, participants received a hyperlink enabling them to access an online survey prepared by the following program https://docs.google.com/ spreadsheets $/ \mathrm{u} / 0$. Participants were required to complete the survey during their next menstruation. The inclusion criteria were to be over eighteen, to have spontaneous menstrual cycles, no known gynecological diseases and no use of oestroprogestinbased products. Women not fulfilling these criteria were excluded from the study, as were postpartum women, lactating mothers and women who had recently experienced miscarriage or abortion. 94 mooncup users were finally recruited.

The independent variables included were age, parity, profession, place of residence, educational level, contraception, length of cycle, length of menstruation and type of mooncup used. The dependent variables were the size of the mooncup used, frequency with which it was emptied, amount of fluid when emptied and menstrual volume. Given the diverse nature of the 11 different types of mooncups used by the participants, the following criteria were used to classify them: light-flow cups (<20cc.), small cups (20-26cc.), large cups (27-33cc.) and "super" cups (>34cc.). In those cases in which the cups were emptied when only stained the criteria adopted was to consider the amount of menstrual fluid to be $2,5 \mathrm{cc}$. In all cases in which cups were emptied when half full the volume was considered to be half the cup's total capacity. When the cups were totally full, menstrual volume was considered to be the full capacity as stated by the manufacturer.

All the data obtained from the variables were introduced in the SPSS ${ }^{2} 20.0$ (SPSS Inc., Chicago, IL, USA) database. Descriptive analysis was performed using the mean, the median and the standard deviation [SD], confidence interval at 95\% [CI95\%], the number of cases [n] and the percentage [\%]. The link between variables was determined using Pearson's linear correlation coefficient. The study protocol was approved on $28^{\text {th }}$ May 2013 by the Commission of Ethics of San Cecilio University Hospital (Granada, Spain). Use of the hyperlink for access to the online survey indicated that users implicitly accepted the conditions of the study.

\section{Results}

A total of 135 women applied to participate in the study, but 30 were excluded as they failed to fulfill the inclusion criteria. 11 of those who were initially admitted did not subsequently fill in the online questionnaire. The study is therefore based on data from 94 women ( $70 \%$ participation). The average age of the sample was 33.21 years (SD 5.90, CI 95\% 32-34.42). In $26 \%$ of cases participants held a postgraduate degree and $23 \%$ had a Bachelor's degree or other undergraduate university diploma. More than half of the sample, 52\% (49 cases), had scientific or intellectual technical or professional careers. Place of residence varied widely amongst participants, although most of the participants recruited were from Spain's two largest cities, Madrid and Barcelona, with 15\% of participants (14 cases) and 7\% (7 participants) respectively. There were also participants from less densely populated areas of the country. With regard to menstrual history, $80 \%$ of the sample had menstrual cycles of 25-33 days with an average length of 4.86 bleeding days (SD 1.05 , CI95\% 4.62-5.10). As for contraceptive methods, 53\% of participants were male condom users, $6 \%$ ( 6 cases) had a copper IUD, and $23 \%$ (22 cases) were using no contraceptive method at the time of the survey. $16 \%$ of participants were nulliparous (62 cases) and $34 \%$ (32 cases) had given birth at least once.

With regard to mooncup use habits, the study reported cups being emptied 1,354 times with an average of 14.4 cups emptied per woman per menstruation (SD 7.43, CI95\% 12.88-15.93), which amounts to an average of 3.04 cups per woman per day (SD 1.43 , CI95\% 2.71-3.37). As far as content was concerned $28 \%$ of cups were totally full when emptied (mean 5.15, SD 3.70, CI95\% 4.29-6.01), 44\% were partially full (mean 6.40, SD 4.05, CI95\% $5.57-7.23$ ) and in $28 \%$ of cases cups were only stained (mean 4.32, SD 2.58, CI95\% 3.77-4.87). Figure 1 reflects emptying habits for each day of menstruation. Based on the capacity of each of the mooncups used, it was possible to estimate that the mean volume of menstrual fluid per woman was $173.20 \mathrm{cc}$ (SD 139.08, CI95\% 144.24-202.17) and that $75 \%$ of participants exceeded the 80cc level of MBL. Figure 2 presents the MBL distribution by age. Clot expulsion rate was $70 \%$. In Table 1 the mean daily menstruation volume is presented. However, if we focus exclusively on the $75 \%$ of participants $(\mathrm{N}=70)$ who emptied the cup when it was totally full, the mean menstrual volume is $110.04 \mathrm{cc}$ (SD 98.01, CI95\% 86.67-133.41), and 47\% of these participants exceeded 80cc MBL. The most widely used mooncups were the low-capacity light flow versions $(37 \%$ cases), followed by large (35\%) whilst small (21\%) and "super" cups $(3 \%)$ were the least used. In total, the mean capacity of all the emptied cups was 22.60cc (SD 7.28, CI95\% 21.08-24.11). Table 2 presents the number of times the participants emptied their cup during the first 5 days of menstruation. 


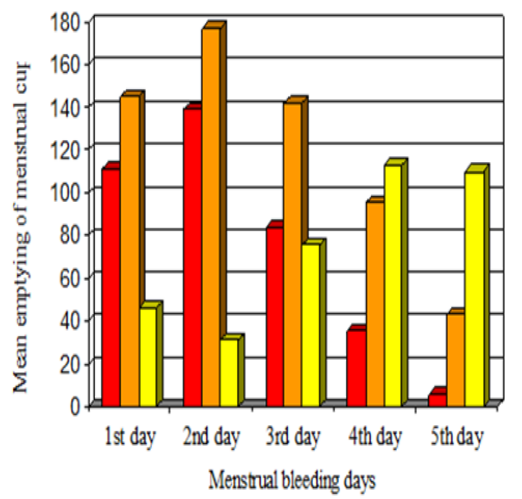

Figure 1: No of mooncups and volume when emptied (total).

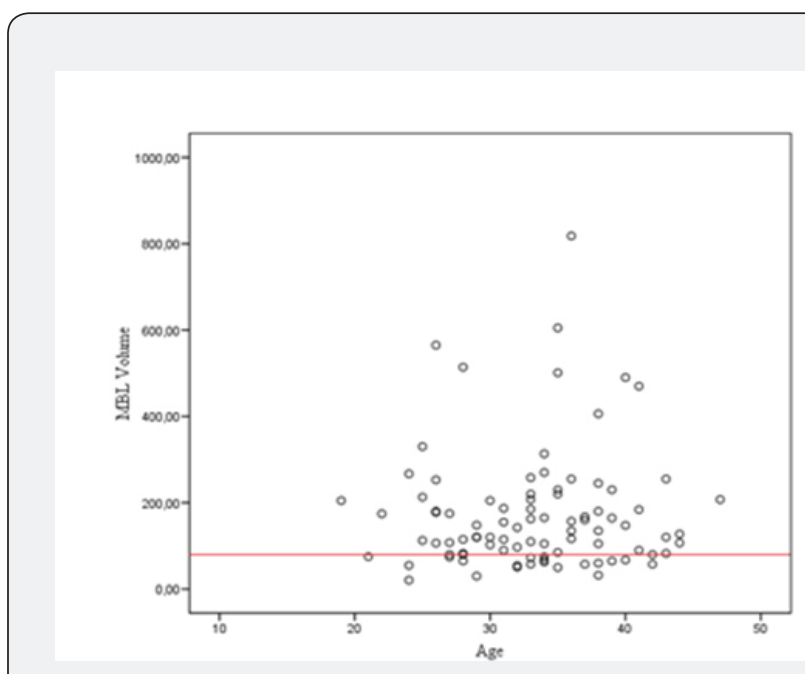

Figure 2: Distribution of $M B L$ volume by age.

Table 1: Estimation of daily MBL.

\begin{tabular}{|c|c|c|c|c|c|}
\hline \multicolumn{7}{|c|}{ Days of menstruation } \\
\hline & Day 1 & Day 2 & Day 3 & Day 4 & Day $\mathbf{5}$ \\
\hline MBL volume (in cc.) & $48.12 \pm 44.84$ & $53.80 \pm 41.54$ & $40.52 \pm 39.15$ & $27.97 \pm 30.46$ & $15.88 \pm 19.47$ \\
\hline \multicolumn{7}{|c|}{ Data represented as mean \pm SD } \\
\hline
\end{tabular}

Table 2: Estimation of daily MBL.

\begin{tabular}{|c|c|c|c|c|c|}
\hline \multicolumn{7}{|c|}{ Days of menstruation } \\
\hline & Day 1 & Day 2 & Day 3 & Day 4 & Day 5 \\
\hline No of Mooncups & 302 & 347 & 302 & 244 & 159 \\
\hline No. of Mooncups/woman & $3.25 \pm 1.77$ & $3.69 \pm 1.93$ & $3.39 \pm 1.80$ & $3.13 \pm 1.64$ & $2.52 \pm 1.49$ \\
\hline \multicolumn{7}{|c|}{ Data represented as mean \pm SD } \\
\hline
\end{tabular}

\section{Discussion}

The use of the mooncup as a tool to estimate Menstrual Blood Loss (MBL) has enabled such estimations to be performed in an objective way and the results suggest that levels may double those considered to be normal or standard for a population of healthy women [5-11]. Until now, that calculation had been based on the PBAC and other, more complex analytical methods. Not all women use the same size of mooncup, so estimations may be slightly higher or lower than real amounts. However, bearing in mind the high number of nulli gravid participants in the study, the predominant trend was to use smaller mooncups, following the manufacturers' indications, which, to a certain extent, would seem to rule out gynecological disease as the cause of high menstrual bleeding. Moreover, frequency of emptying differed amongst the participants. Therefore, when participants indicated that the cup was half full or merely stained this may have led to differences between the estimated amount and the real amount of menstrual fluid lost. However, if we focus exclusively on the mooncups that were emptied when they were totally full, the menstrual volume figures are high. Furthermore, the fact that the emptying rate was estimated as being higher than 3 times per day leads us to believe that the MBL estimation is fairly accurate.
Regarding daily emptying frequency we have seen that the highest number of totally full cups corresponded to the second day of menstruation, in accordance with our previous findings and with the results of other authors, although it must be stressed that indirect estimation methods were used [4,12-14]. This leads us to believe that these new findings in this study are likely to be reasonably accurate. Some authors have asserted that women discard some 14 sanitary pads during menstruation [10]. Many women are alarmed by the perception that menstrual bleeding is accompanied by the expulsion of clots of different sizes. The prevalence of clot expulsion during menstrual bleeding and its frequency is approximately $55 \%$, depending on age $[12,15,16]$. In our sample, up to $70 \%$ of participants perceived clot expulsion, to a greater extent on day $2(52 \%)$, which is a much higher figure than that offered by other authors $[4,17]$. In summary, we have seen that MBL and clot expulsion rate in our sample could be double the figures described in literature on this subject.

\section{Limitations}

Our study may be considered to have certain biases, such as a sample composed of highly educated participants who were predominantly resident in large urban areas. This may have facilitated their access to information about the mooncup 


\section{Global Journal of Reproductive Medicine}

and meant that they had the resources and capacity to access online questionnaires. Women who were suffering from some gynecological condition were excluded from the study which means that we could not analyze the possible causes of HMB which, in any case, was not one of the study's objectives. Perhaps one of the study's strengths is that it offers a novel method for a clinical approach to the diagnosis of menstrual bleeding problems, unlike other previously used techniques.

\section{Conclusions and Recommendations}

\section{Conclusion}

In summary, the menstrual mooncup has facilitated realistic estimation of menstrual volume in healthy women, making it an easy and accessible tool for use in clinical practice.

\section{Recommendation}

It would be recommendable to design more extensive population studies that would allow us to determine whether MBL has currently increased in healthy women.

\section{Acknowledgement}

We are grateful to all the women who generously participated in the study despite the inconvenience of having to access the online questionnaire on a daily basis.

\section{References}

1. Howard C, Rose CL, Trouton K, Stamm H, Marentette D, et al. (2001) FLOW (finding lasting options for women): multicentre randomized controlled trial comparing tampons with menstrual cups. Can Fam Physician 57(6): e208-215.

2. Stewart K, Greer R, Powell M (201) Women's experience of using the Mooncup. J Obstet Gynaecol 30(3): 285-287.

3. Mason L, Laserson KF, Oruko K, Nyothach E, Alexander KT, et al. (2015) Adolescent schoolgirls' experiences of menstrual cups and pads in rural western Kenya: a qualitative study. Waterlines 34(1): 15-30.

4. Martos García R (2016) Application of a questionnaire about the menstrual cycle and non-hormonal contraceptive use in Andalusian women Granada: Universidad de Granada.

5. Munro MG (2012) Classification of menstrual bleeding disorders. Rev Endocr Metab Disord 13: 225-234.
6. Fraser IS, Critchley HO, Broder M, Munro MG (2011) The FIGO recommendations on terminologies and definitions for normal and abnormal uterine bleeding. Semin Reprod Med 29(5): 383-390.

7. Sociedad Española de Ginecología y Obstetricia (2013) Sangrado menstrual abundante (SMA) (Actualizado 2013). Prog Obstet Ginecol 56(10): 535-546.

8. Diaz A, Laufer MR, Breech LL, American Academy of Pediatrics, Committee on Adolescence, American College of Obstetricians and Gynecologists, Committee on Adolescent Health Care(2006) Menstruation in girls and adolescents: using the menstrual cycle as a vital sign. Pediatrics 118(5): 2245-2250.

9. Higham JM, Obrien PMS, Shaw RW (1990) Assessment of menstrual blood-loss using a pictorial chart. Br J Obstet Gynaecol 97(8): 734-749.

10. Gudmundsdottir BR, Hjaltalin EF, Bragadottir G, Hauksson A, Geirsson RT, et al. (2009) Quantification of menstrual flow by weighing protective pads in women with normal, decreased or increased menstruation. Acta Obstet Gynecol Scand 88(3): 275-279.

11. Zakherah MS, Sayed GH, El-Nashar SA, Shaaban MM (2011) Pictorial blood loss assessment chart in the evaluation of heavy menstrual bleeding: diagnostic accuracy compared to alkaline hematin. Gynecol Obstet Invest 71(4): 281-284.

12. Sánchez J, Andrab S, Bercaw JL, Dietrich JE (2012) Quantifying the PBAC in a Pediatric and Adolescent Gynecology Population. Pediatr Hematol Oncol 29(5): 479-484.

13. Mihm M, Gangooly S, Muttukrishna S (2011) The normal menstrual cycle in women. Anim Reprod Sci 124(3-4): 229-236.

14. Lukes AS, Baker J, Eder S, Adomako TL (2012) Daily menstrual blood loss and quality of life in women with heavy menstrual bleeding. Womens Health (Lond) 8(5): 503-511.

15. Santos IS, Minten GC, Valle NC, Tuerlinckx GC, Silva AB, et al. (2011) Menstrual bleeding patterns: A community-based cross-sectional study among women aged 18-45 years in Southern Brazil. BMC Womens Health 11(1): 1-8.

16. Parker MA, AE Sneddon AE, Arbon P (2010) The menstrual disorder of teenagers (MDOT) study: determining typical menstrual patterns and menstrual disturbance in a large population based study of Australian teenagers. BJOG 117(2): 185-192.

17. Warner PE, Critchley HOD, Lumsden MA, Campbell-Brown M, Douglas A, et al. (2004) Menorrhagia I: Measured blood loss, clinical features, and outcome in women with heavy periods: A survey with follow-up data. Obstet Gynecol 190(5): 1216-1223.

Your next submission with Juniper Publishers
will reach you the below assets
- Quality Editorial service
- Swift Peer Review
- Reprints availability
- E-prints Service
- Manuscript Podcast for convenient understanding
- Global attainment for your research
- Manuscript accessibility in different formats
( Pdf, E-pub, Full Text, Audio)
- Unceasing customer service
Track the below URL for one-step submission
https://juniperpublishers.com/online-submission.php

